\title{
Target Segmentation Algorithm Based on the Discrete Energy
}

\section{Functional}

\author{
Zhi Ping Wan* \\ Xinhua College of Sun Yat-sen University, Guangzhou, 510520, China \\ wzp888_0@126.com
}

Keywords: Segmentation algorithm; Discretization; Energy functional; Object extraction.

\begin{abstract}
Existing segmentation algorithm has the segmentation problem that is vulnerable to background noise and low accuracy. In order to address this problem, the discrete Technology is used in this research. And the target segmentation algorithm is based on discrete energy functional. It is effectively reduce the complexity and accuracy, which using discrete processing of the regional items and contour curve. Experimental results indicate that article algorithm can be maintained a good target extraction in the case of the target and the background which gradually increasing similarity. And the preponderance of article algorithm can be found through correctness and time-consuming experiment.
\end{abstract}

\section{Introduction}

Image segmentation algorithm in computer vision has always been an important research focus. It is divided into many small regions to extracting a target signal based on certain rules. So it was plays a vital role in image processing. As we known, nowadays the researchers was put forward a lot of target segmentation algorithms [1-3]. For example, the segmentation algorithm based on threshold [4], the segmentation algorithm based on histogram [5], and the segmentation algorithm based on clustering Method, etc. Although it get the target signal was quickly, but its segmentation accuracy and anti-jamming capabilities are unsatisfactory. Although people was put forward to many new target segmentation algorithm, but there were cannot solve this problem.

In recent years, some researchers were proposed the target segmentation algorithm based on the Energy Functional. This approach can largely improve the accuracy of the algorithm. But it still exist the problems that were distortion and interference in the case of the complex background and the target similarity of the background. In response to this phenomenon, this paper presents target segmentation algorithm based on the discrete energy functional. Reduce the complexity and optimization structure of the algorithm was using the discrete theorem. It was making the object extraction processes converted to the Mathematical optimization process. That was beneficial to improve the accuracy of object segmentation algorithm. And it can be always kept higher resolution results under different background environment.

\section{Energy functional}

Traditional energy functional algorithm get the higher segmentation accuracy was using the similarity, smoothness and roughness of the edges, etc. In the process of Mathematical transformation, researchers express a function by creating the image fidelity term, regular items and curved contours length term C. Function expression is as follows. 
Finally get the expression: $F(u, C)=\iint_{\Omega}\left|u(x, y)-u_{0}(x, y)\right|^{2} d x d y+\alpha \iint_{\Omega \backslash C}|\nabla u(x, y)|^{2} d x d y+\beta \int_{C} d \sigma$

In order to obtain good approximation results, people was using piecewise constant and variational methods to improve this algorithm. Expression is as follows.

$F\left(C, c_{1}, c_{2}\right)=\lambda_{1} \iint_{\Omega i n}\left|u(x, y)-c_{1}\right|^{2} d x d y+\lambda_{2} \iint_{\Omega o u t}\left|u(x, y)-c_{2}\right|^{2} d x d y+\eta|C|$

Therein $c_{1}$ and $c_{2}$ was the background mean and objectives mean of both sides of the curved profile C. And $\eta 、 \lambda_{1} 、 \lambda_{2}$ was the constant. In order to facilitate the following discrete processing, this paper was optimization of formula (1), which combine with the Heavside function $H(x)$ and Dirac function $\delta(x)$.Function expression is as follows.

$\left.F\left(\varphi, c_{1}, c_{2}\right)=\lambda_{1} \iint_{\varphi \geq 0}\left|u(x, y)-c_{1}\right|^{2} H(\varphi(x, y)) d x d y+\lambda_{2} \iint_{\varphi(0)}\left|u(x, y)-c_{2}\right|^{2}(1-H(\varphi(x, y))) d x d y\right)+\eta|C|$

By using gradient descent to solve $c_{1}$ and $c_{2}$, And in accordance with $c_{1} 、 c_{2}$ Iterative update to get the value of minimize. $\quad c_{1}(\varphi)=\frac{\iint_{\Omega} u(x, y) H(\varphi(x, y)) d x d y}{\iint_{\Omega} H(\varphi(x, y)) d x d y} ; \quad c_{2}(\varphi)=\frac{\iint_{\Omega} u(x, y)(1-H(\varphi(x, y))) d x d y}{\iint_{\Omega}(1-H(\varphi(x, y))) d x d y}$

At finally, we can get an approximate solution: $u(x, y)=c_{1} H(\varphi(x, y))+c_{2} H(\varphi(x, y))$

\section{Article algorithm}

Discrete processing of the Area items. This paper analyzes the probability of the target image. So we was defined the probability of target and background. Make the target section satisfied $\mathrm{f}^{(x, y) \geq \lambda}$, and background section satisfied $f(x, y)<\lambda$ [6-7]. Set up $(i, j)$ as image coordinate points, and referred to the reference point.

So we can get the dimensional joint probability density: $P_{i j}=f_{i, j} / N$

Therein $i, j=0,1,2, \cdots, L-1$, and Satisfy the conditions $\sum_{\mathrm{i}=0}^{L-1} \sum_{j=0}^{L-1} P_{\mathrm{i}, j}=1$.

I.e. Target probability: $P_{b}=\sum_{i=s+1}^{L-1} \sum_{\mathrm{j}=t+1}^{L-1} P_{\mathrm{i} j} ;$ Background probability: $P_{f}=\sum_{i=0}^{s} \sum_{\mathrm{j}=0}^{t} P_{\mathrm{i} j}$

The study found that mean can effectively reflect the relationship between the target and background. So that it was beneficial to improve the algorithm's ability to distinguish between target and background. Therefore, we can get the mean function, which was using the gap measure theorem. Target Mean: $\mu_{\mathrm{b}}(t)=\left(\mu_{\mathrm{bi}}, \mu_{b j}\right)^{T}=\left(\frac{\sum_{i=0}^{s} \sum_{\mathrm{j}=0}^{t} i P_{\mathrm{ij}}}{P_{b}(t)}, \frac{\sum_{i=0}^{s} \sum_{\mathrm{j}=0}^{t} j P_{\mathrm{ij}}}{P_{b}(t)}\right)^{T}$

Background mean: $\mu_{\mathrm{f}}(t)=\left(\mu_{\mathrm{fi}}, \mu_{f j}\right)^{T}=\left(\frac{\sum_{i=s+1}^{L-1} \sum_{\mathrm{j}=t+1}^{L-1} i P_{\mathrm{i} j}}{P_{f}(t)}, \frac{\sum_{i=s+1}^{L-1} \sum_{\mathrm{j}=t+1}^{L-1} j P_{\mathrm{i} j}}{P_{f}(t)}\right)^{T}$

So article algorithm was introduction of the target and background mean function. Make the formula (3) and (4) Substituted into the formula (2). As follows: 
$F\left(c_{1}, c_{2}, P\right)=\lambda_{1} \sum_{P_{b}}\left|u(i, j)-c_{1}\right|^{2} \mu_{b}(t)+\lambda_{2} \sum_{P_{f}}\left|u(i, j)-c_{2}\right|^{2} \mu_{f}(t)+\eta|C|(\varphi=0)$

Therein, $\quad c_{1} 、 c_{2}$ Discrete processing were $c_{1}=\frac{\sum_{P_{b}} u(i, j) \mu_{b}(t)}{\sum_{P_{b}} \mu_{b}(t)} ; \quad c_{2}=\frac{\sum_{P_{f}} u(i, j) \mu_{f}(t)}{\sum_{P_{f}} \mu_{f}(t)}$

Discretization of the contour curves. Hypothesis that each pixel corresponds to a node, and relationship between adjacent pixels to form a side. So we can calculate the approximate length of the contour, which according to cross edge of the contour curves.

Step 1: Defines the value of the length term $\eta$. In the image processing, If different pixel image by using a fixed global constant to distinguish, would be leading to the phenomenon of over-segmentation or segmentation omissions. So this paper defines an adaptive shape functions: $\eta=\mathrm{e}^{-\left(P_{b}(t)-P_{f}(t)\right)^{2}}$. And article algorithm was using it to measure the relationship between each pixel in the target and the background.Therein, definition of $e_{k}$ value range is Satisfied of [0,1]. It was benefit to reduce redundant calculations.

Step 2: Determining the value of $\omega_{k}$. Set up coordinate system and make the noise figure is 1 .

So the function expression of the distance between the field is $\mathrm{e}_{k}=\frac{1 \cdot\left|\sin \theta_{k}\right|+1\left|\cos \theta_{k}\right|}{2}$

Introduction of Direction Factor: $e_{0}=e_{\pi / 4}=e_{\pi}=e_{3 \pi / 2}=\frac{1}{2} ; e_{\pi / 2}=e_{3 \pi / 4}=e_{5 \pi / 4}=e_{7 \pi / 4}=\sqrt{2}$

I.e. $\omega_{1}=\omega_{3}=\omega_{5}=\omega_{7}=\frac{\pi}{16}, \omega_{2}=\omega_{4}=\omega_{6}=\omega_{8}=\frac{\pi}{8 \sqrt{2}}$

Step 3: Calculate $n_{k}$. Above all, Depending on the nature of the detection function $d(b, f)$. We can determine the nature of the region was comparison of the Background mean and objective mean.

When $d(b, f)$ Is close to 0, Said it was contour areas, 1.e. $d(b, f)=\frac{\left|\mu_{\mathrm{b}}(t)-\mu_{\mathrm{f}}(t)\right|^{2}}{N \mathrm{um}(\mathrm{n})}$

Therein, $\operatorname{Mim}(n)$ represents the number of pixels in the region.

Step 4: Generating the discretization of the contour curve. Get a new contour curve length that was Combination of the above formula $\omega_{k}$ and formula (6). So $\mid$ C $\mid=\frac{1}{2} \sum_{P_{b}, P_{f} \in \Omega} \omega_{k} \cdot \frac{\left|\mu_{\mathrm{b}}(t)-\mu_{\mathrm{f}}(t)\right|^{2}}{N \mathrm{~m}(\mathrm{n})}$

The Discretization formula of the length items is: $\eta|C|=\frac{\mathrm{e}^{-\left(P_{b}(t)-P_{f}(t)\right)^{2}}}{2} \sum_{P_{b}, P_{f} \in \Omega} \omega_{k} \cdot \frac{\left|\mu_{\mathrm{b}}(t)-\mu_{\mathrm{f}}(t)\right|^{2}}{h \mathrm{um}(\mathrm{n})}$ $F\left(x_{1}, \cdots, x_{n}\right)=\lambda_{1} \sum_{P_{b}}\left|u(i, j)-c_{1}\right|^{2} \mu_{b}(t)+\lambda_{2} \sum_{P_{f}}\left|u(i, j)-c_{2}\right|^{2} \mu_{f}(t)+\frac{\mathrm{e}^{-\left(P_{b}(t)-P_{f}(t)\right)^{2}}}{2} \sum_{P_{b}, P_{f} \in \Omega} \omega_{k} \cdot \frac{\left|\mu_{\mathrm{b}}(t)-\mu_{\mathrm{f}}(t)\right|^{2}}{N \mathrm{~mm}(\mathrm{n})}$

In summary, article algorithm can get the minimization model discretization of energy, which based on above several Discretization of operations. 


\section{Simulation experiments}

In order to verify the effectiveness of method, using the simulation environment is MATLAB 2014a. The compilation environment configuration of article algorithm was shown in Table 1.

Table 1 Compilation environment configuration data table

\begin{tabular}{cc}
\hline Hardware & Data \\
\hline CPU & i5-4460 (Quad-Core) \\
Memory & 8 GBytes
\end{tabular}

Graphics Discrete graphics 2GB ，Nvidia GT620

Hard disk Hitachi HTS545050A7E380

Compare with other algorithms. In order to test the ability of the object extraction, this paper was selection the similarity of the target and background, and there similarities were gradually increased. Experimental results show that article algorithm can kept the image clarity in the case of complex background.
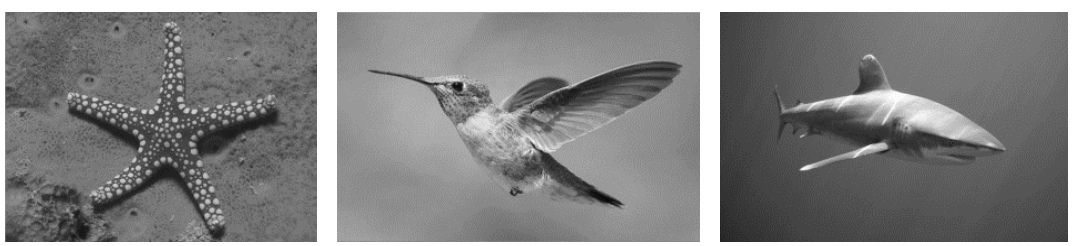

Figure, 1 Image to be processed
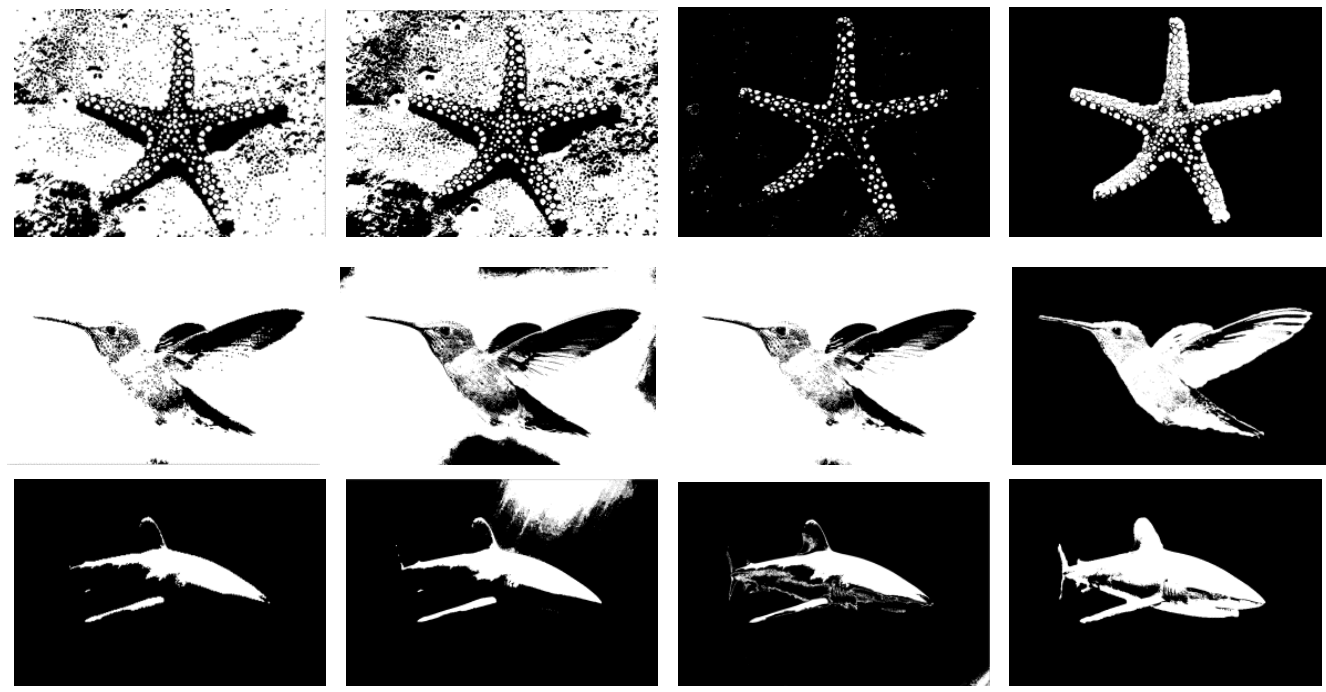

(a) Literature Algorithm [8] (b) Literature Algorithm [9] (c) Literature Algorithm [10] (d) Article algorithm Figure. 2 The simulation map of each algorithm

Compare with the Run time and accuracy. In order to better evaluate the article algorithm, we were contrast with the Run time and accuracy of segmentation effect.

Table 2 Running time of the algorithm

\begin{tabular}{ccccc}
\hline \multirow{2}{*}{ Image } & \multicolumn{4}{c}{ Running time /s } \\
& $\begin{array}{c}\text { Literature } \\
\text { Algorithm[8] }\end{array}$ & $\begin{array}{c}\text { Literature } \\
\text { Algorithm[9] }\end{array}$ & $\begin{array}{c}\text { Literature } \\
\text { Algorithm[10] }\end{array}$ & $\begin{array}{c}\text { Article } \\
\text { algorithm }\end{array}$ \\
\hline Starfish & 2.11 & 3.21 & 2.98 & 2.21 \\
Hummingbird & 2.38 & 3.41 & 3.23 & 2.47 \\
Shark & 2.01 & 2.70 & 2.62 & 2.83 \\
\hline
\end{tabular}


Table 3. Correct rate of each segmentation algorithms

\begin{tabular}{ccccc}
\hline \multirow{2}{*}{ Image } & \multicolumn{4}{c}{ Correct rate /\% } \\
& Literature & Literature & Literature & Article \\
\hline \hline Starfish & Algorithm[8] & Algorithm[9] & Algorithm[10] & algorithm \\
\hline Hummingbird & 73.81 & 89.88 & 87.77 & 95.21 \\
Shark & 69.89 & 76.14 & 89.76 & 93.24 \\
\hline
\end{tabular}

By combining the data in Table 1 and Table 2, we can be found that the accuracy of the algorithm has remained at $94.77 \%$. And it has advantages of effectively object segmentation. And that can maintain accuracy in the case of the highest similarity. But in segmentation process, observe the operation time can be seen that article algorithm and other algorithms have same time-consuming. The average consumption of time was 2.50 .

\section{Summary}

Existing algorithms has the poor treatment effect in the case of complex background and objectives high similarity with background. In response to this phenomenon, we were proposed a target segmentation algorithm that has the characteristics of High reliability and accuracy. And in order to obtain the optimal target segmentation results, it was using the discretization definition to optimization of conventional energy functional. Experimental results show that the experimental results were consistent with the expected results. And it has advantage of the strong anti-jamming capability and high precision of Segmentation.

\section{References}

[1] Nashat S, Abdullah A, Abdullah MZ. Unimodal thresholding for Laplacian-based Canny-Deriche filter [J]. PATTERN RECOGNITION LETTERS. 2012, 33(11): 1269-1286.

[2] Ilk HG, Jane O, Ilk O. The effect of Laplacian filter in adaptive unsharp masking for infrared image enhancement [J]. INFRARED PHYSICS \& TECHNOLOGY. 2011,54(5): 427-438.

[3] He QH, Zhang ZX. A new edge detection algorithm for image corrupted by White-Gaussian noise $[\mathrm{J}]$. AEU-INTERNATIONAL JOURNAL OF ELECTRONICS AND COMMUNICATIONS. 2007,61(8): 546-550

[4] Ranjani JJ, Thiruvengadam SJ. Fast threshold selection algorithm for segmentation of synthetic aperture radar images [J]. IET RADAR SONAR AND NAVIGATION. 2012,6(8): 788-795.

[5] Yang YF, Wang YP. Simulated annealing spectral clustering algorithm for image segmentation [J].JOURNAL OF SYSTEMS ENGINEERING AND ELECTRONICS. 2014, 25(03): 514-522.

[6] Avraham T, Lindenbaum M. Esaliency (Extended Saliency): Meaningful Attention Using Stochastic Image Modeling [J]. IEEE TRANSACTIONS ON PATTERN ANALYSIS AND MACHINE INTELLIGENCE. 2010, 32(4): 693-708.

[7] Chen Q, Zhao L, Lu J, Kuang G, Wang N, Jiang Y. Modified two-dimensional Otsu image segmentation algorithm and fast realization [J]. IET Image Processing. 2012,6(04): 426-433.

[8] Zhang P, Liang YM, Chang SJ, Fan HL. Kidney segmentation in computed tomography sequences based on energy minimization [J]. Acta Physica Sinica. 2013,62(20):1-6.(in chinese)

[9] Xu SJ. Algorithm of minimizing local region energy for image segmentation [J]. Journal of Xi'an Jiaotong University. 2011, 45(8):7-12.

[10]Qi G, Liang X, Hu H, Zhi HW. An active contour model driven by anisotropic region fitting energy for image segmentation [J]. Digital Signal Processing. 2013, 35(4): 791-796. 